\title{
Seminal Vesicle Involvement by Carcinoma In Situ of the Bladder: Clonal Analysis Using Next-Generation Sequencing to Elucidate the Mechanism of Tumor Spread
}

\author{
Hyun Sik Park, MD \\ Hyun Bin Shin, MD' \\ Myung-Shin Lee, MD, PhD² \\ Joo Heon Kim, MD, PhD 3 \\ Seon-Young Kim, $\mathrm{PhD}^{4}$ \\ Jinsung Park, MD, PhD'
}

${ }^{1}$ Department of Urology, Eulji University Hospital, Eulji University School of Medicine, Daejeon, Departments of ${ }^{2}$ Microbiology and Immunology and ${ }^{3}$ Pathology, Eulji University School of Medicine, Daejeon, ${ }^{4}$ Personalized

Genomic Medicine Research Center,

Korea Research Institute of Bioscience $\mathcal{E}$

Biotechnology, Daejeon, Korea

Correspondence: Jinsung Park, MD, PhD

Department of Urology, Eulji University

Hospital, Eulji University School of Medicine,

95 Dunsanseo-ro, Seo-gu, Daejeon 35233, Korea

Tel: 82-42-611-3533

Fax: 82-42-259-1111

E-mail: jspark.uro@gmail.com

Received January 2, 2020

Accepted March 17, 2020

Published Online March 19, 2020
We present a rare case of urothelial carcinoma in situ (CIS), which invades the prostate and seminal vesicle (SV). A 70-year-old man underwent transurethral resection of bladder (TURB), and the pathologic examination revealed multiple CIS. Although the patient received intravesical bacillus Calmette-Guerin (BCG) therapy following TURB, recurrence of CIS was confirmed in the bladder and left distal ureter at 3 months following BCG. Radical cystectomy was performed due to BCG-refractory CIS. Microscopically, CIS was found throughout the mucosa of the bladder, left ureter, prostatic duct, and both SVs. Next-generation sequencing revealed significant differences in tumor clonality between bladder and SV CIS cells. Among 101 (bladder CIS) and 95 (SV CIS) somatic mutations, only two were shared, and only one gene (ARHGAP23) was common exon coding region gene. In conclusion, multicentric genetic changes, in line with the field-cancerization effect, may result in $\mathrm{SV}$ involvement by $\mathrm{CIS}$ of the bladder.

\section{Key words}

Urinary bladder neoplasms, Seminal vesicle involvement, Carcinoma-in-situ, High-throughput nucleotide sequencing, Clonality

\section{Introduction}

Bladder cancer (BC) is the second most common cancer of the genitourinary tract worldwide [1]. Approximately $75 \%$ of patients with $B C$ present with non-muscle invasive $B C$ which is either confined to the mucosa (stage Ta and carcinoma in situ [CIS]) or the submucosa (stage T1) [2]. Non-muscle invasive $B C$ is characterized by frequent recurrence, and muscleinvasive BC often metastasizes to regional or distant lymph nodes and distant sites such as the bone, lung, and liver. However, the seminal vesicle (SV) involvement of urothelial carcinoma is uncommon [3-5]. Hypothetically, two distinct patterns have been suggested to explain how primary BC extends to the SVs: One is direct invasion through the bladder wall and perivesical fat, which occurs in most cases, while the other pattern is pagetoid mucosal spread of urothelial carcinoma, which is uncommon $[5,6]$.
Specifically, CIS of the SVs has been previously reported by few studies [5-8], always in association with multifocal CIS of the bladder, prostate, and ureter. Although several possibilities including pagetoid mucosal spread, tumor cell implantation, and de novo development of urothelial carcinoma $[5,6]$ have been suggested with regard to the mechanism of the SV involvement by CIS, clonal analysis of the tumor cells has not been analyzed yet. Clonal analysis of the bladder and SV CIS cells may be helpful to elucidate the mechanism by which CIS invades the SV. Next-generation sequencing (NGS) is a technology of DNA sequencing for genomic research, and new knowledge may be obtained by employing NGS in the field of $\mathrm{BC}$ [9]. In this study, we present a very rare case of bladder CIS, which did not respond to bacillus Calmette-Guerin (BCG) therapy and invaded the prostatic duct and SV. For the first time, we also report results of tumor clonal analysis from CIS cells of bladder and SV using NGS. 


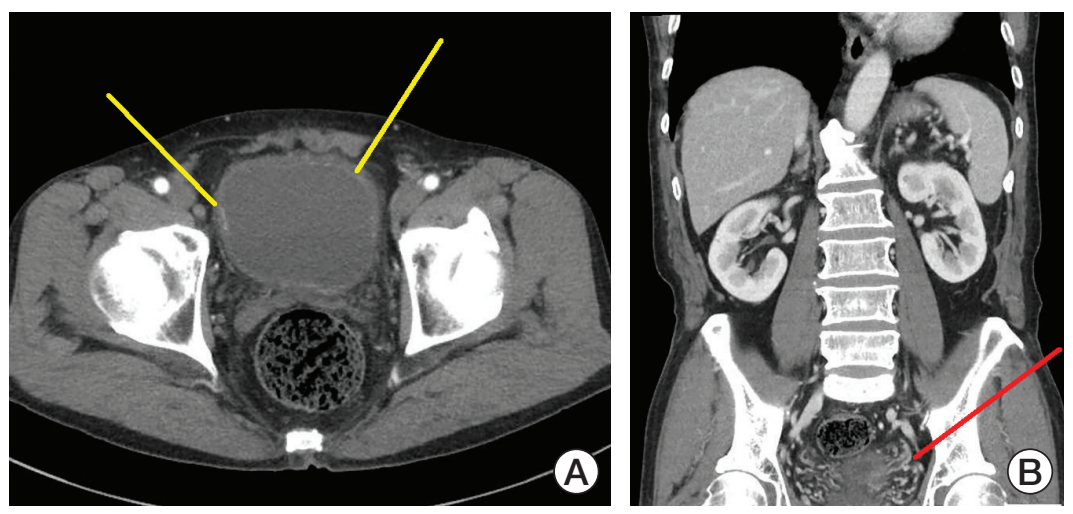

Fig. 1. Computed tomography scan shows multiple enhancing lesion at bladder mucosa (yellow lines) (A) and segmental enhancement of the left distal ureter (red line) (B).

\section{Case Report}

\section{Case}

A 70-year-old man visited the Eulji University Hospital with gross hematuria, with no specific medical history. As diagnostic work-ups for hematuria, cystoscopy showed a wide spread of velvety lesions on posterior bladder wall. The computed tomography (CT) scan revealed focal enhancing lesion at the right lateral and left anterolateral wall of the bladder and segmental enhancement of the left distal ureter (Fig. 1). For pathologic diagnosis of the lesions on bladder and left distal ureter, retrograde-pyelography (RGP), diagnostic ureteroscopy (URS), and transurethral resection of bladder (TURB) were performed. The RGP showed ureterovesical junction narrowing and proximal ureter kinking. No definite mass was observed in the left distal ureter, in diagnostic URS. Therefore, random biopsies were performed in left distal ureter, where segmental enhancement was observed on CT scan. All velvety lesions in the bladder were resected using a bipolar resectoscope. The pathologic findings depicted multifocal urothelial CIS in the bladder and left distal ureter. At postoperative 2 weeks, the patient was treated with 6 weeks of intravesical BCG. At 3 months after TURB, follow-up CT showed enhancing lesions at multiple bladder mucosa and left distal ureter again. The patient underwent diagnostic URS and TURB again. Diagnostic URS showed left distal ureter obstruction with a mass-like lesion, and multiple biopsies of ureter lesion were performed, while TURB was also performed for the newly developed multiple velvety lesions in the bladder. The pathologic findings showed multiple urothelial CIS in the bladder, but no malignancy of ureter. Since the tumor was considered to be BCG-refractory CIS of bladder, the patient underwent robot-assisted radical cystectomy with ileal neobladder. After radical cystectomy, the patient recovered without any specific complications and did not undergo adjuvant chemotherapy. Up to 24 months after cystectomy, there was no evidence of recurrence in fol- low-up CT scans and urine cytology examinations.

The microscopic findings of cystectomy specimens showed urothelial CIS throughout the mucosa of the bladder, left ureter, prostatic urethra with prostatic ductal extension and multifocal stromal invasion, ejaculatory ducts, and SV (Fig. 2). Tumor cells revealed distinct nuclear membranes and relatively abundant eosinophilic cytoplasm. The nuclei of tumor cells were large, hyperchromatic, markedly pleomorphic and angular with irregular contours, coarse chromatin, prominent nucleoli, and frequent mitoses. The tumor cells showed pagetoid spread into the prostatic acini and ducts, neighboring ejaculatory ducts and the mucosa of the SV. These pagetoid spread were mainly observed between the intact overlying epithelium and underlying basement membrane of prostatic ducts and/or acini and SV, thereby resulting in complete replacement of ducts and acini by tumor cells. In immunohistochemical staining, the tumor cells in the urinary bladder as well as the prostatic ducts and SV were seen to be positive for cytokeratin 20 and p53, but negative for CD44 and prostate-specific antigen.

Whole exome sequencing was performed to analyze the genomic differences between CIS cells of the bladder and SVs. A total amount of $1.0 \mu \mathrm{g}$ genomic DNA per sample was used as an input material for the DNA library preparation using formalin-fixed, paraffin-embedded samples obtained from bladder and SV lesions under light microscopy by an experienced uro-pathologist (J.H.K.). Blood sample was used as a normal reference for the two tumor samples. Sequencing libraries were generated using the Agilent SureSelect Human All Exon V7 kit (Agilent Technologies, Santa Clara, $\mathrm{CA}$ ), following the manufacturer's recommendations, and index codes were added to each sample. The average depths for each sequenced data were 109.33 (blood), 136.06 (bladder CIS), and 117.50 (SV CIS), respectively. We used the MuTect [10] to detect somatic single nucleotide variants (SNVs), and the Strelka to detect somatic small insertions and deletions (InDels), and copy number variations (CNVs) in the tumor 

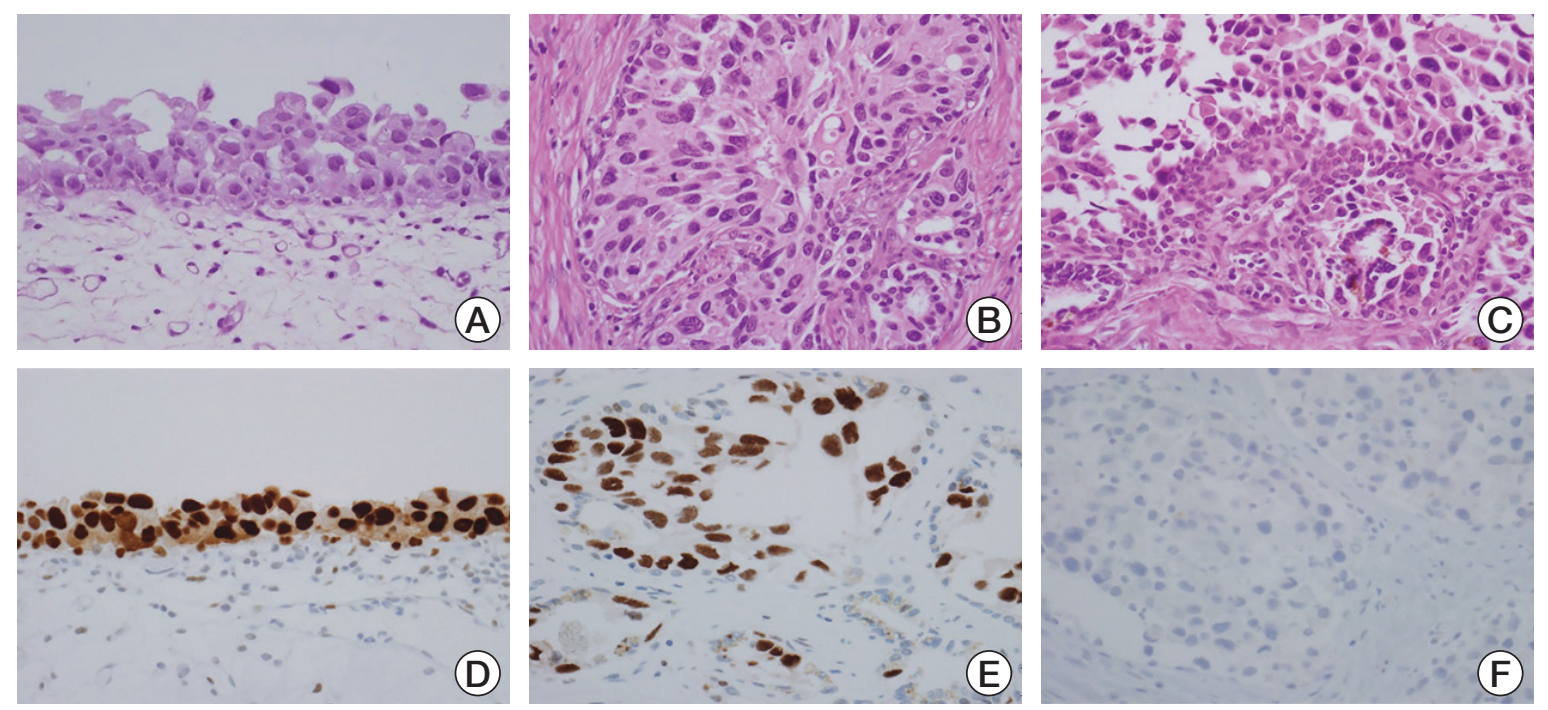

Fig. 2. Microscopic findings of radical cystectomy specimen. (A) Urothelial carcinoma in situ (CIS) is observed in the urinary bladder, in which mucosal epithelium is covered by the highly anaplastic tumor cells (H\&E staining, $\times 400)$. (B) The prostatic acini and ducts are also involved by neoplastic urothelial cells (H\&E staining, $\times 400)$. (C) The mucosa of the seminal vesicle shows pagetoid spread of the tumor cells (H\&E staining, $\times 400)$. (D) In the urothelial CIS of the bladder, p53 was highly expressed on the nuclei of tumor cells $(\times 400)$. (E) p53 was highly expressed in pagetoid spreading tumor cells of the seminal vesicle $(\times 400)$. (F) Prostate-specific antigen was not expressed in the tumor cells in the ducts of prostate $(\times 400)$.

Table 1. Common somatic single nucleotide variants which were shared by the bladder and seminal vesicle carcinoma in situ lesions

\begin{tabular}{lcccccccc} 
Chromosome & Startp & Endp & $\begin{array}{c}\text { Ref_ } \\
\text { allele }\end{array}$ & $\begin{array}{c}\text { Var_- } \\
\text { allele }\end{array}$ & $\begin{array}{c}\text { VAF of } \\
\text { bladder }\end{array}$ & $\begin{array}{c}\text { VAF of } \\
\text { seminal vesicle }\end{array}$ & Category & Gene \\
11 & 68549472 & 68549472 & $\mathrm{C}$ & $\mathrm{T}$ & 0.448 & 0.273 & Intronic & CPT1A \\
17 & 36636008 & 36636008 & $\mathrm{C}$ & $\mathrm{T}$ & 0.250 & 0.278 & Exonic & ARHGAP23 \\
\hline
\end{tabular}

VAF, variant allele frequency.

samples. SNVs and InDels with variant allele frequency $(\geq 0.1)$ and sequencing read $(\geq 10)$ were used for further analysis. Mutations were defined as genes with a frequency of less than $1 \%$ in three human genomic databases such as 1000 Genomes, Exome Aggregation Consortium (ExAC) and exome sequencing project. We also excluded SNVs frequently observed in Korean population by using KRGDB of 1,722 samples (http:// coda.nih.go.kr/coda/KRGDB/index. jsp). We found only two common SNVs among $101 \mathrm{SNVs}$ of bladder CIS and 95 SNVs of SV CIS lesions (Table 1). Among the two SNVs which were shared by the bladder and SV tumor cells, only one gene (Rho GTPase activating protein 23 [ARHGAP23]) lie in exonic coding region. Notably, we found significant differences in the genomic changes between bladder and SV tumor cells, including the SNVs (S1 and S2 Tables), the InDels (S3 and S4 Tables), and the CNVs (S5 and S6 Tables).

\section{Ethical statement}

The study protocol was approved by the Institutional
Review Board of the Eulji University Hospital (No. 201912-011). Written informed consent was obtained from our patient after explaining the present study.

\section{Discussion}

Multifocality is an important feature of urothelial carcino$\mathrm{ma}$, frequently occurring in the $\mathrm{BC}$, renal pelvis, and ureter tumors [11]. The concept of "field change" and "a monoclonal origin" has been proposed with regard to the multifocal nature $[12,13]$. The monoclonal theory suggests that multiple tumors occur from an intraluminal seeding or intra-epithelial migration of single malignant transformed urothelial cells [14]. In contrast, the field change theory describes that exposure of carcinogens may lead to independent genetic change at different sites of the urinary tract [15]. Many previous studies [12-15] were carried out to analyze the clonality of multifocal urothelial carcinoma, but at present there is no consensus whether multifocal lesions have a monoclonal ori- 
gin or an independent origin.

Meanwhile, the incidence of SV involvement by urothelial carcinoma is reported to be approximately $3 \%$ in radical cystectomy cases [4-6]. However, SV involvement by CIS is very rare [5-8]. Notably, no prior study analyzed the mechanism of the SV involvement, but some previous studies [5-8] have suggested that bladder CIS cells of monoclonal origin spread to SV. For example, two U.S. studies have hypothetically suggested the implantation or intramucosal metastasis of monoclonal tumor cells [5] or intraepithelial (pagetoid) spread of bladder CIS to SV [6] as a mechanism of the SV involvement. Similarly, a Korean study suggested CIS involvement of SV by the pagetoid spread of bladder CIS rather than the de novo development of separate SV CIS, based on microscopic features [8].

In line with the hypothetical mechanism, our pathological findings and immunohistochemical staining results support the notion of pagetoid mucosal extension of bladder CIS to prostatic ducts and SV. However, of note, our whole exome sequencing analyses results indicate that CIS cells of SV were quite distinct from bladder CIS cells in terms of clonality. For example, among somatic SNVs frequently detected in BC, TP53 (one of tumor suppressor genes) and KDM6A (one of most commonly mutated, chromatin-modifying genes in BC) mutations were present in bladder CIS, but absent in SV CIS (S1 and S2 Tables). Meanwhile, ZDHHC21, PGGHG, and USP7 mutations, which were reported to be associated with BC, were present in SV CIS only, not in bladder CIS. Notably, only one gene (ARHGAP23) was common exon coding region gene, supporting our findings. Thus, in contrast to the circumstantial evidence suggested by previous studies $[5,6,8]$, our results based on direct clonal analysis indicate that multiclonal oncogene activation or loss of tumor suppressor genes may be responsible for the formation of separate CIS lesions in SVs with more or less malignant behavior, depending on the type of genetic change [13]. Interestingly, our NGS analysis showed that majority of somatic variants are non-coding mutations (such as intronic, ncRNA_exonic, ncRNA_intronic, etc.) while clinical significance of those mutations is largely unknown (S1 and S2 Tables). Thus, clinical significance of those mutations needs further investigation with more samples, although SV involvement by CIS of the bladder is extremely rare. Integrating our results, we believe that SV involvement by CIS may not occur by hypo- thetical pagetoid spread of bladder CIS cells, but independent tumor progression in SVs through field-cancerization effect.

The prognostic significance of the SV involvement by bladder CIS remains unclear due to its infrequency [8]. Although several studies have noted that mucosal spread to SV should be a separate subcategory owing to its better prognosis compared to direct SV invasion $[4,5]$, more cases are needed to define the prognosis of patients with SV involvement by CIS. Meanwhile, a prior study raised the possibility of inadequate pathological sampling as a reason of the uncommon occurrence of SV involvement by BC in surgical pathology laboratories [6]. Thus, the adequate evaluation of SV involvement in radical cystectomy specimens is needed. Importantly, because all reported cases including our study had multifocal CIS in urinary tract (bladder, ureter, prostatic duct, and $\mathrm{SV})$ and our data support multicentric genetic changes in urinary tract, we need to carefully monitor synchronous CIS development in remnant urinary tracts with regular $\mathrm{CT}$ scan and urine cytology.

To our knowledge, our study is the first report to analyze the tumor clonality between bladder and SV CIS using NGS to elucidate the mechanism of tumor spread into the SV. Integrating our results, we believe that multicentric genetic changes, in line with the field-cancerization effect, may result in SV involvement by CIS of the bladder. Although, the pagetoid spread of CIS in SV is uncommon, the clinician must be aware of the possibility of SV involvement by CIS, specifically in patients with multifocal CIS lesions. Further studies are needed to determine clinical implications of SV involvement by CIS.

\section{Electronic Supplementary Material}

Supplementary materials are available at Cancer Research and Treatment website (https://www.e-crt.org).

\section{Conflicts of Interest}

Conflict of interest relevant to this article was not reported.

\section{Acknowledgments}

This research was supported by the Basic Science Research Program through the National Research Foundation of Korea (NRF), funded by the Ministry of Education (2019R1I1A3A01060913).

\section{References}

1. Global Burden of Disease Cancer Collaboration; Fitzmaurice C, Allen C, Barber RM, Barregard L, Bhutta ZA, et al. Global, regional, and national cancer incidence, mortality, years of life lost, years lived with disability, and disability-adjusted life-years for 32 cancer groups, 1990 to 2015: a systematic analysis for the global burden of disease study. JAMA Oncol. 2017;3: 524-48.

2. Babjuk M, Bohle A, Burger M, Capoun O, Cohen D, Comperat 
EM, et al. EAU guidelines on non-muscle-invasive urothelial carcinoma of the bladder: update 2016. Eur Urol. 2017;71:44761.

3. Daneshmand S, Stein JP, Lesser T, Quek ML, Nichols PW, Miranda G, et al. Prognosis of seminal vesicle involvement by transitional cell carcinoma of the bladder. J Urol. 2004;172: 81-4.

4. Volkmer BG, Kufer R, Maier S, Bartsch G Jr, Bach D, Hautmann $\mathrm{R}$, et al. Outcome in patients with seminal vesicle invasion after radical cystectomy. J Urol. 2003;169:1299-302.

5. Montie JE, Wojno K, Klein E, Pearsall C, Levin H. Transitional cell carcinoma in situ of the seminal vesicles: 8 cases with discussion of pathogenesis, and clinical and biological implications. J Urol. 1997;158:1895-8.

6. Ro JY, Ayala AG, el-Naggar A, Wishnow KI. Seminal vesicle involvement by in situ and invasive transitional cell carcinoma of the bladder. Am J Surg Pathol. 1987;11:951-8.

7. Jakse G, Putz A, Hofstadter F. Carcinoma in situ of the bladder extending into the seminal vesicles. J Urol. 1987;137:44-5.

8. Choi SY, Lee HC, Song HG, Kim WJ, Lee OJ. Seminal vesicle involvement by urothelial carcinoma in situ of the bladder with mucosal spread pattern: a case report. Korean J Urol. 2012;53:368-70.
9. Pietzak EJ, Bagrodia A, Cha EK, Drill EN, Iyer G, Isharwal S, et al. Next-generation sequencing of nonmuscle invasive bladder cancer reveals potential biomarkers and rational therapeutic targets. Eur Urol. 2017;72:952-9.

10. Cibulskis K, Lawrence MS, Carter SL, Sivachenko A, Jaffe D, Sougnez C, et al. Sensitive detection of somatic point mutations in impure and heterogeneous cancer samples. Nat Biotechnol. 2013;31:213-9.

11. Kiemeney LA, Witjes JA, Heijbroek RP, Verbeek AL, Debruyne FM. Predictability of recurrent and progressive disease in individual patients with primary superficial bladder cancer. J Urol. 1993;150:60-4.

12. Sidransky D, Frost P, Von Eschenbach A, Oyasu R, Preisinger $\mathrm{AC}$, Vogelstein B. Clonal origin of bladder cancer. N Engl J Med. 1992;326:737-40.

13. Harris AL, Neal DE. Bladder cancer: field versus clonal origin. N Engl J Med. 1992;326:759-61.

14. Habuchi T, Takahashi R, Yamada H, Kakehi Y, Sugiyama T, Yoshida O. Metachronous multifocal development of urothelial cancers by intraluminal seeding. Lancet. 1993;342:1087-8.

15. Jones TD, Wang M, Eble JN, MacLennan GT, Lopez-Beltran A, Zhang S, et al. Molecular evidence supporting field effect in urothelial carcinogenesis. Clin Cancer Res. 2005;11:6512-9. 